\title{
Events in 2015
}

\section{Announcement}

The Korean Council of Science Editors announces the schedule of the events in 2015. Out of them, the 2nd Asian Science Editors' Conference and Workshop (August 20-22, 2015) will be hell in Hanoi University of Science and Technology, Hanoi, Vietnam. Theme of the conference and workshop is 'How to add local scientific journals to international databases.' There will be preconference workshop in August 20, 2015. One and half day is allocated for the conference. In the afternoon, August 22, 2015, there will be a tour in Hanoi city. The precise schedule and the call for papers will be propagated up to end of February 2015 through homepage of the Council of Asian Science Editors available from: http://asianeditor.org/.

The 15th Editors' Workshop (March 2-3, 2015) will be held in the Korean Federation of Science and Technology Societies, Seoul. This workshop will deal with Journal Article Tag Suite extensible markup language.

In May 2015, there will be launch of manuscript editor certificate program by the Korean Council of Science Editors. This program will be organized by Ms. Hye-Min Cho, Chair of Committee on Manuscript Editing. It is the first trial in Korea to train and certify the performance of manuscript editors.

The 16th Editors' Workshop (July 2-3) will be held in Seoul. It will be workshop for reviewers and authors.

The 18th Editors' Workshop (December 3-4, 2015) is for novice editors. The program will consist of basic knowledge and skill for novice editor who are new editors or have an experience as editors less than three years.

Precise schedule and registration of above workshops were or will be available from: http:// www.kcse.org/.

\begin{tabular}{|c|c|c|c|c|c|c|}
\hline & January & February & March & April & May & June \\
\hline Science Editing (twice/yr) & & Vol.2 No.1 (20) & & & & \\
\hline Newsletter (4 times/yr) & & & №. $13(31)$ & & & No. 14 (30) \\
\hline \multicolumn{7}{|l|}{ International conference } \\
\hline Editors' workshop & & & $\begin{array}{c}\text { 15th Editors' } \\
\text { Workshop (2-3) }\end{array}$ & & & \\
\hline $\begin{array}{l}\text { Manuscript editors' } \\
\text { training \& workshop }\end{array}$ & $\begin{array}{c}\text { Basic } \\
\text { Manuscript } \\
\text { Editing } \\
(15,22,29)\end{array}$ & $\begin{array}{c}\text { Basic } \\
\text { Manuscript } \\
\text { Editing } \\
(5,12,26)\end{array}$ & $\begin{array}{c}\text { Basic } \\
\text { Manuscript } \\
\text { Editing } \\
(5,12)\end{array}$ & & $\begin{array}{l}\text { Manuscript } \\
\text { editors } \\
\text { certificate } \\
\text { program } \\
\text { (DTBD) }\end{array}$ & \\
\hline $\begin{array}{l}\text { Publication ethics } \\
\text { forum \& workshop }\end{array}$ & & $\begin{array}{c}\text { 9th } \\
\text { Publication } \\
\text { Ethics Forum } \\
\text { (12) }\end{array}$ & & & & $\begin{array}{c}\text { 10th } \\
\text { Publication } \\
\text { Ethics } \\
\text { Workshop (18) } \\
\text { 11th } \\
\text { Publication } \\
\text { Ethics } \\
\text { Workshop (19) }\end{array}$ \\
\hline
\end{tabular}




\section{science editing}

\begin{tabular}{|c|c|c|c|c|c|c|}
\hline & July & August & September & October & November & December \\
\hline Science Editing (twice/yr) & & Vol.2 No.2 (20) & & & & \\
\hline International conference & & $\begin{array}{l}\text { 2nd Asian } \\
\text { Science Editors' } \\
\text { Conference } \\
\text { and Workshop } \\
\text { (20-22) }\end{array}$ & & & & \\
\hline Editors' workshop & $\begin{array}{c}\text { 16th Editors' } \\
\text { Workshop (2-3) }\end{array}$ & & & & & $\begin{array}{c}\text { 18th Editors' } \\
\text { Workshop (3-4) }\end{array}$ \\
\hline $\begin{array}{l}\text { Manuscript editors' } \\
\text { training \& workshop }\end{array}$ & $\begin{array}{c}\text { Advanced } \\
\text { Manuscript } \\
\text { Editing } \\
(10,14,16,23)\end{array}$ & & $\begin{array}{l}\text { 17th } \\
\text { Manuscript } \\
\text { Editors' } \\
\text { Workshop } \\
\text { (3-4) }\end{array}$ & & & \\
\hline $\begin{array}{l}\text { Publication ethics } \\
\text { forum \& workshop }\end{array}$ & & & & & & \\
\hline
\end{tabular}

DTBD, date to be determined. 